\title{
Facilitating Difficult Discussions in STEM
}

Gregory R. Goldsmith, Brenna M.G. Gormally, Rebecca Green, Aaron W. Harrison, Brian A. Hoover, Kenjiro Quides, Zachary Thammavongsy, Shana R. Welles, Bingjie Zhang \& Kelsey M.

Gray

Grand Challenges Initiative, Chapman University, Orange, CA 92866 USA

Correspondence to: G.R. Goldsmith, One University Drive, Schmid College of Science and Technology, Chapman University, Orange, CA 92866 USA; +1-714-516-5881; goldsmit@chapman.edu

\begin{abstract}
Discussion can be an important and powerful tool in efforts to build a more diverse, equitable, and inclusive future for STEM. However, facilitating discussions on complex and uncomfortable issues, like racism and sexism, can feel daunting. We outline a series of steps that can be used in offices, laboratories, and classrooms to facilitate productive discussions that empower everyone to listen, contribute, learn, and ultimately act to transform STEM.
\end{abstract}

\section{Introduction}

It should be no secret that science, technology, engineering and mathematics continue to have serious problems related to diversity, equity, and inclusion (Calisi et al., 2018; Hughes, 2018; Lee et al., 2020; NSF, 2019). Given the increased awareness surrounding issues such as racism (Barber et al., 2020; Miriti, 2020) and gender bias (Handley et al., 2015; Helmer et al., 2017), we have a renewed opportunity to make meaningful changes in STEM and to make it a place where everyone feels welcomed, valued, and supported in pathways to success. Many of us in STEM may be more open and eager than ever to talk about these issues in our offices, classrooms, and laboratories. What if we lack the tools to facilitate these discussions? After all, we are trained to hold pipettes, write code, and teach evolutionary theory, not dig deep into difficult, complex and often uncomfortable issues.

Discussing difficult issues is important and compelling. When we allow it to, it exposes us to new perspectives and leads to new ideas. It helps us recognize and investigate our biases and assumptions. It allows us to identify differences and commonalities. It encourages us to be better listeners and communicators. It promotes empathy. Discussion can be a powerful means of affecting change (Brookfield and Preskill, 2012).

In the undergraduate science and engineering education program we teach in, we believe that engaging in discussion every week is a fundamental part of enhancing our students' ability to develop a STEM identity and use STEM to make the world around them a better place. Through these discussions, we also begin to realize a more diverse, equitable, and inclusive future in 
STEM. We strive to create a space for each and every person to provide their perspectives on difficult issues, question how they know what they know, and ultimately upend the status quo by asking new questions and devising innovative ways to think about the world.

\section{Setting Up For Success}

What do we need to do to facilitate successful discussions? Our approach is drawn from many great resources developed both within and outside of the fields of STEM (e.g., Bell et al., 2016). We have applied discussion primarily in the context of helping our students explore issues ranging from gender bias in peer review to racial and socioeconomic disparities in exposure to air pollution (i.e., what may be referred to as socio-scientific issues; Sadler, 2011), but we describe our approach in a way intended to be applicable in many different contexts.

We start by collaboratively establishing guidelines to foster community, build buy-in, and create a safe space. Common guidelines may include listening respectfully and without interruption, allowing everyone to speak who wishes to do so by some established method (e.g. raising their hand), asking questions for clarification and minimizing assumptions, focusing discussion on ideas rather than on the individuals bringing up the idea, and not asking anyone to speak on behalf of all people sharing a particular identity (Brookfield and Preskill, 2012). It is important that everyone has explicit permission to make mistakes, be given grace when they do so, and provided room to grow. This is no different from the expectations you should be afforded when learning how to do a complicated protocol in the lab for the first time. These guidelines may seem evident, but we find that they can easily be forgotten amidst a difficult conversation.

We then build a shared sense of purpose for the discussion and anchor the discussion in materials that bring evidence to bear on the topic. For instance, we may all agree that the purpose of the discussion is to understand how and why educational opportunities and attainment in STEM varies among different identities (e.g., Bernard and Cooperdock, 2018). We assign reading on the topic and ask students to provide brief written reflections about the materials before they arrive, so that everyone has time to consider the information and their own interpretations. This also provides the opportunity for everyone to contribute, even if they do not wish to speak out loud.

With a shared sense of purpose, established ground rules and materials that serve as the anchor for discussion, we often begin by asking everyone to consider a sentence completion exercise, such as "What struck me the most as I read/watched/listened to this was..." To reduce the hesitation to speak first, this can be coupled with a brief "think-pair-share" approach before bringing it back to the whole group (Kaddoura, 2013). We avoid beginning discussion with any form of summary or lecture, as this tends to shift the tone from participatory to didactic (see Box 1 for example questions to use in discussion).

Box 1. A collection of helpful phrases that can be used while facilitating discussions. Wherever possible, it may be useful to direct these to everyone participating in the discussion, rather than singling out an individual. 


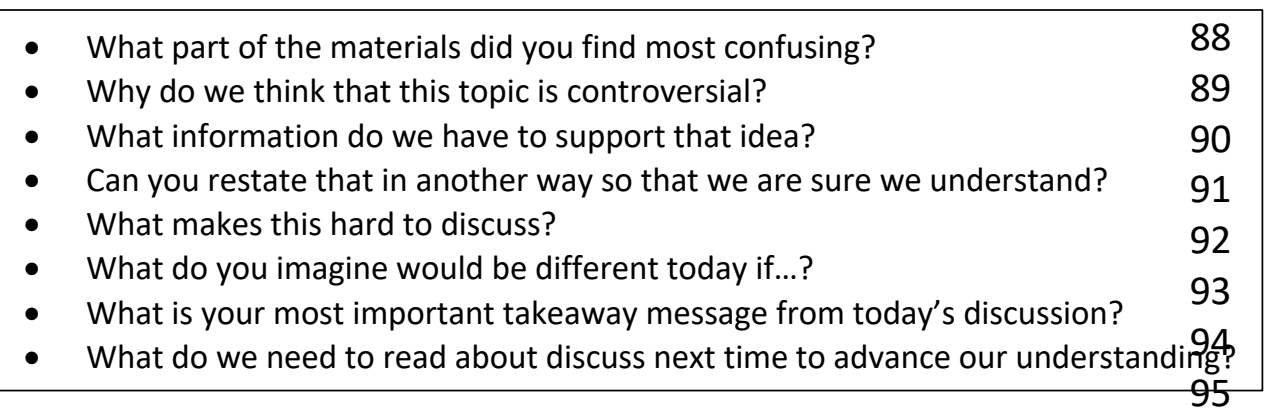

96

When we facilitate, we are responsible for practicing careful listening, both to what people are saying and to the other cues they are providing through their choice of words, tone of voice, and their body language (Sorensen, 2017). We also hold primary responsibility for making sure that everyone feels their ideas have been given due attention. This can be as simple as repeating the idea back and asking for the person to confirm that you understood correctly, or thanking the person for being willing to share their idea. Repeating or thanking the person for sharing is a valuable means of maintaining a space for all ideas, even if we do not agree with them. An example discussion guideline might be that everyone summarize the previous speaker's statement before proceeding with their own statement as a way of ensuring active listening (Brookfield and Preskill, 2012). While facilitating, it is important to recognize that silence is $\mathrm{OK}$ and to resist the temptation to fill the void. Explicitly providing time to think can reduce the extent to which the discussion is monopolized by a small number of individuals and amplify voices that may not otherwise be heard.

Ultimately, as facilitators, we strive to model the behavior we wish to see in those around us. These behaviors include following and enforcing the guidelines, listening carefully and asking thoughtful questions, keeping the conversation grounded in the materials, guarding against misinformation, and ensuring that everyone is heard. It will not always go as planned.

\section{Effectively Addressing Controversy}

How do we respond when controversy inevitably arises? When necessary, we immediately return to the ground rules established. For instance, if someone raises their voice and makes an accusation directed at an individual, we reply by reminding everyone in the room (rather than targeting an individual) that everyone agreed on a respectful dialogue focused on the ideas. We may also choose to explicitly address the emotions (e.g., reticence, defensiveness, or anger) that we perceive to help everyone consider why they are experiencing those emotions. Remember that not all personalities and cultures will respond in the same way to conflict (LeBaron, 2003).

It is important to acknowledge the difference between a controversial comment and an offensive comment. Controversy can aid effective discussions (Johnson et al., 2000). However, when someone says something particularly offensive, you may choose to pause the conversation. For instance, we can say "OK, let's all pause for a second and get a cup of coffee or use the restroom." This provides you with an opportunity to decide whether or not you 
believe that the conversation can continue in a constructive manner, which involves both your own comfort and ability, as well as the comfort of those around you. If an offensive statement has been made, then it may not be true that everyone will be ready and able to continue learning from one another at that time. If you decide to conclude the discussion for the day, be sure to follow up with both the individuals directly involved and with the whole group by email in order to facilitate reflection.

If you choose to proceed with discussion following a controversial or offensive comment, you may ask everyone to write about what they are thinking and how they are feeling for a few minutes to increase the likelihood of additional constructive discussion. Before writing begins, ask clarifying questions and restate the issue at hand to address misunderstandings. For instance, we often observe strong reactions when people make statements that lack specificity (e.g., too many pronouns lead to generalizations about a specific group). In deconstructing an overtly controversial statement, it is also important to separate statements based on opinions and values from statements based on evidence. We may ask "what evidence would we need to support this idea?" This forces us to confront underlying assumptions and can help re-ground the discussion. By framing the question to everyone, we avoid singling out a particular individual.

We plan for both the conclusion of the discussion and next steps. We encourage everyone to reflect on what they have learned, what went well in the discussion, and what could be improved in the future. We try to do so both in the group setting, as well as individually. In practice, this can be as simple as having everyone write down three takeaway messages on notecards that you summarize in a follow-up email (Ruland and Ahern, 2007). We also make a plan for the next steps (e.g., further discussion or a specific action) that we wish to take. Finally, we reach out to those who were particularly affected by the discussion and share additional resources with them.

\section{Do Not Expect Perfection}

Like all of the skills we learn in STEM, learning to effectively facilitate difficult discussions will not happen overnight. It comes with practice. It comes with the recognition that we cannot (and should not) control every last moment. And like most things in science, it will not always turn out the way we had hoped. However, if we aim to address issues of diversity, equity, and inclusion in science and engineering, then we need to create equitable and inclusive spaces for 167 a diversity of ideas to be shared, supported, and ultimately acted upon. 


\section{References (5 Recommended Further Readings in Bold Font)}

Barber PH, Hayes TB, Johnson TL, Márquez-Magaña L, 10,234 signatories. 2020. Systemic racism in higher education. Science 369:1440.2-1441. doi:10.1126/science.abd7140

Bell LA, Goodman DJ, Ouellett ML. 2016. Design and FacilitationTeaching for Diversity and Social Justice. Routledge. pp. 55-94.

Bernard RE, Cooperdock EHG. 2018. No progress on diversity in 40 years. Nature Geoscience 11:292-295. doi:10.1038/s41561-018-0116-6

Brookfield SD, Preskill S. 2012. Discussion as a Way of Teaching: Tools and Techniques for Democratic Classrooms. John Wiley \& Sons.

Calisi RM, et al. 2018. Opinion: How to tackle the childcare-conference conundrum. Proc Natl Acad Sci USA 115:2845-2849. doi:10.1073/pnas.1803153115

Handley IM, Brown ER, Moss-Racusin CA, Smith JL. 2015. Quality of evidence revealing subtle gender biases in science is in the eye of the beholder. PNAS 112:13201-13206. doi:10.1073/pnas.1510649112

Helmer M, Schottdorf M, Neef A, Battaglia D. 2017. Gender bias in scholarly peer review. eLife 6:e21718. doi:10.7554/eLife.21718

Hughes BE. 2018. Coming out in STEM: Factors affecting retention of sexual minority STEM students. Science Advances 4:eaao6373. doi:10.1126/sciadv.aao6373

Johnson DW, Johnson RT, Tjosvold D. 2000. Constructive controversy: The value of intellectual oppositionThe Handbook of Conflict Resolution: Theory and Practice. Hoboken, NJ, US: Jossey-Bass/Wiley. pp. 65-85.

Kaddoura M. 2013. Think Pair Share: A Teaching Learning Strategy to Enhance Students' Critical Thinking. Educational Research Quarterly 36:3-24.

LeBaron M. 2003. Culture and Conflict. Beyond Intractability. Accessed 29 September 2020 at https://www.beyondintractability.org/essay/culture-conflict

Lee MJ, Collins JD, Harwood SA, Mendenhall R, Huntt MB. 2020. "If you aren't White, Asian or Indian, you aren't an engineer": racial microaggressions in STEM education. IJ STEM Ed 7:48. doi:10.1186/s40594-020-00241-4

Miriti MN. 2020. The Elephant in the Room: Race and STEM Diversity. BioScience 70:237-242. doi:10.1093/biosci/biz167

NSF. 2019. Women, Minorities, and Persons with Disabilities in Science and Engineering (No. Special Report NSF 19-304). Alexandria, Va.

Ruland JP, Ahern NR. 2007. Transforming Student Perspectives Through Reflective Writing. Nurse Educator 32:81-88. doi:10.1097/01.NNE.0000264328.56039.1b

Sadler TD. 2011. Socio-scientific Issues in the Classroom: Teaching, Learning and Research. Springer Science \& Business Media.

Sorensen MS. 2017. I Hear You: The Surprisingly Simple Skill Behind Extraordinary Relationships. Autumn Creek Press. 